\title{
Nach dem Staats in die Südsee
}

\section{Helen Laura Moser}

MMed; Resident Fellow, Icahn School of Medicine at Mount Sinai, New York

\author{
Nachdem ich im Sommer 2016 mein Staatsexamen erfolgreich absolviert hatte und \\ meine erste Stelle erst im Januar 2017 begann, buchte ich einen Flug nach Honiara, \\ der Hauptstadt der Salomonen, einer Inselgruppe im Südpazifik. Mein Ziel war, in \\ einem Provinzspital die Ärzte etwas zu entlasten. Die Salomonen kannte ich be- \\ reits aus meiner Zeit als Unterassistentin. Dazumal arbeitete ich fast drei Monate \\ auf der Orthopädie im National Referral Hospital (NRH). Dort lernte ich die lokale \\ Sprache Pijin zu verstehen, etwas zu sprechen sowie das Land zu schätzen.
}

In Honiara angekommen, wurde mir mit einem einzi-
gen Telefonanruf bei Dr. Grace, die mit ihrem Mann
Dr. Arnold das Kirakira Hospital führt, alles Weitere
organisiert. Rund eine Woche später landete ich nach
drei abgesagten Flügen in der Makira-Ulawa-Provinz,
auch bekannt als Bananenprovinz der Salomonen.
Über 40 Bananensorten wachsen hier. Mit der Ambu-
lanz, welche gerade einen Patient zum Flugzeug ge-
bracht hatte, ging es zum Guesthouse. Dort traf ich auf

\section{Résumé}

Mes études terminées et mon examen d'Etat en poche, je décidai de retourner dans le Pacifique sud. Je pouvais ainsi apporter mon soutien aux médecins locaux et les décharger un peu de leurs lourdes tâches. J'étais heureuse de pouvoir renouer des contacts avec ces terres que je connaissais d'un précédent séjour à Honiara, capitale des lles Salomon. Cette fois je décidai de dédier ce nouveau séjour à des activités médicales en faveur des patients de l'hôpital provincial de Kirakira. Ma connaissance du terrain et de la langue locale (Pijin) me permirent de m'investir pleinement et de manière intense pour des activités variées.

Les moyens pour établir les diagnostics étaient limités et il fallait trouver des solutions simples et originales. Ma capacité à improviser et mon habilité manuelle me furent de précieuses aides. Le pays dispose de peu de moyens mais lorsqu'ils sont bien utilisés ils permettent d'extraordinaires résultats. Les indigènes sont patients et reconnaissants du réconfort apporté par le médecin. Ma mission s'est terminée peu après un tremblement de terre important (7,8 sur l'échelle de Richter) alors que j'étais l'unique médecin sur l'île. J'ai quitté un hôpital heureusement resté intact et fonctionnel mais qui doit pour quelques temps encore se passer de médecin.

Je garde un souvenir inoubliable des lles Salomon et je compte bien y retourner.

Les médecins/étudiants intéressés peuvent consulter le site de l'association «Medizin im Südpazifik» www.hermannoberli.ch pour obtenir des informations relatives à de potentielles missions. vier Medizinstudentinnen und Dr. James Finn, den Initiator der Beziehungen zwischen der Makira-Provinz und der australischen Bond University (www.strong islandfoundation.com.au). Doch diese reisten bereits zwei Tage später wieder ab. So war ich die meiste Zeit die einzige Weisshäutige im Dorf und eine der wenigen in der ganzen Provinz.

Das Spital ist für 50000 Menschen zuständig und liegt in Kirakira, einem verschlafenen 2500-Seelen-Dorf an der Nordküste von Makira, direkt neben meinem Guesthouse. Sobald die Sonne untergegangen ist, kann man nichts mehr machen. Doch wenn man untertags nicht gerade arbeitet, kann man einen der beiden Strände mit einem kurzen Spaziergang erreichen. Zudem gibt es perfekte Bedingungen für eine morgendliche Joggingrunde.

Eine stündige Bootsfahrt entfernt gibt es wunderschöne Inseln mit weissen, palmengesäumten Stränden und badewannenwarmem Wasser.

Am ersten Abend bekam ich eine Spitalführung und wurde den Patienten vorgestellt. Die zwei Ärzte waren froh und dankbar, dass ich kam, um sie etwas zu entlasten. Sie sind 24/7 auf Abruf.

Glücklicherweise wurde mir auch das einzige GratisWiFi der Insel gezeigt. Die Verbindung war meist so schlecht, dass ein Mail senden oft 5-10 Minuten in Anspruch nahm und nur je nach Wetterlage möglich war.

\section{Meine Arbeit}

Das Spital ist täglich sehr gut besucht. Man merkt, dass wir weit und breit das einzige Spital sind und viele Patienten von kleinen Buschkliniken aus der ganzen Provinz geschickt werden.

Glücklicherweise arbeitet die Pflege ziemlich selbständig und behandelt die Patienten oftmals selber. Leider 
fast immer mit Antibiotika, ob indiziert oder nicht. Wir Ärzte schauen uns nur die akuteren Fälle an und diejenigen, bei denen die Pflege nicht weiter weiss. Oftmals sind das Patienten mit chronischen Leiden oder auch Kinder. Zudem sind die Ärzte für die Untersuchung von Gewaltopfern im Rahmen von polizeilichen Ermittlungen zuständig.

Kleinere Prozeduren wie Abszessspaltungen, Debridements oder auch Frakturrepositionen werden unter Lokalanästhesie oder Ketamin durchgeführt. Egal ob Tropenmedizin, HNO, Gynäkologie, Pädiatrie oder Orthopädie, an einem normalen Tag sieht man fast alles. Von meinem ersten Tag an wurde ich voll eingesetzt: Visite am Morgen z.T. mit einem der anderen Ärzte sowie ganztägig Sprechstunde mit vielen kleinen Eingriffen im Outpatient Department. Am ersten Tag war ich schon ab Mittag auf mich alleine gestellt und entfernte

\section{Viele Patienten kamen spät, manche zu spät.}

Fremdkörper aus Nasen und nähte riesige Skalpverletzungen. Die Patientin lag auf einer Liege mitten im Outpatient Department, umgeben von vielen Menschen. Ihr Kopf war mit einem blutigen Tuch bedeckt und ich wusste im ersten Moment nicht, ob sie noch lebte. Unter dem blutigen Tuch befand sich eine riesige, klaffende Kopfschwartenverletzung. Glücklicherweise sah der freiliegende Schädelknochen intakt aus. Die Patientin sei durch einen Streit mit einer anderen Frau von der Leiter gefallen und mit dem Kopf auf einen Stein aufgeschlagen. Danach sei sie eine lange Zeit bewusstlos gewesen. Im OP versorgte ich die Wunde zusammen mit einer der Studentinnen, welche doch ziemlich überrascht über die zähe dunkle melanesische Haut war. Sie hatte ihre Probleme mit dem Nähen. Neurologisch war die Patientin glücklicherweise unauffällig.

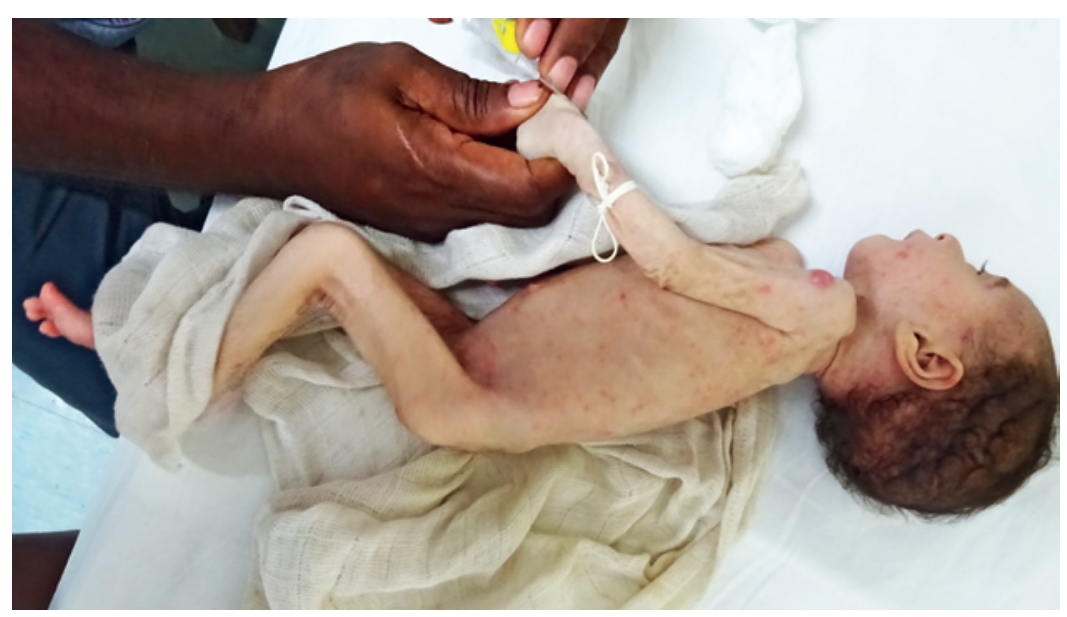

Abbildung 1: Legen eines i.v. Zugangs bei einem mangelernährten Kind (1,9kg).

\section{Salomonen}

Beim Human Development Index (HDI), einem Wohlstandsindikator der Vereinten Nationen, der BIP, Lebenserwartung und Bildungsniveau beinhaltet, lagen die Salomonen 2014 irgendwo gegen Ende noch hinter Ruanda und Angola und knapp vor Uganda und Sudan, also in bester Gesellschaft mit anderen Entwicklungsländern (Schweiz Platz 3).

Stürze von Bäumen und von Häusern sind häufige Unfälle, v.a. auch bei Kindern.

\section{Die Herausforderungen}

Viele Patienten kamen spät, manche zu spät. Oftmals sahen wir einen entzündeten Blinddarm erst, wenn dieser bereits rupturiert war und eine Peritonitis verursacht hatte. So musste man innert kürzester Zeit reagieren, um den Patienten mit dem nächsten Linienflug in die Hauptstadt zu verlegen, damit er eine Überlebenschance hat. Normalerweise kümmerte sich Dr. Arnold um den Papierkram. War er gerade nicht erreichbar, kam es auch vor, dass ich mit einem Überweisungsschreiben für das NRH und einem netten Brief für den Piloten zum Büro der Solomon Airlines laufen musste, damit der Patient mitgenommen wurde.

Die Arbeit war sehr abwechslungsreich und auch herausfordernd. Ich wurde mit jeglichen Symptomen einer Malariaerkrankung, mit TBC, mit dem sich aus der

\section{Von meinem ersten Tag an wurde ich voll eingesetzt.}

Hauptstadt ausbreitenden Denguefieber und vielen pädiatrischen Problemen konfrontiert. Da mir z.T. das Wissen fehlte, musste ich immer wieder Bücher, Antibiotika- oder WHO-Richtlinien konsultieren. Ich entwickelte jedoch rasch ein Gefühl, welche Patienten stationär behandelt werden mussten oder welche nach Hause geschickt werden konnten. Immer zu berücksichtigen war auch, wie lange ihr Heimweg war. Mehrstündige Schifffahrten, Fussmärsche oder Truckfahrten waren die Regel.

Glücklicherweise hatten wir ein Ultraschall- und ein Röntgengerät. Doch auf die Frage, ob es einen vergrösserten Appendix gab, kam ein Blatt mit der Vermessung der Leber, der Niere und des Uterus zurück. Informationen, die mir nicht wirklich in der Diagnostik weiterhalfen. Auch war der Mann, der die Röntgen durchführte, oftmals abwesend. So wartete ich Stunden, um zu wissen, wie schlimm die Fraktur nun wirklich war. So wurde ein Patient für ein Röntgen auch kurzerhand stationär, nur weil das Boot oder der Truck schon weg war. 


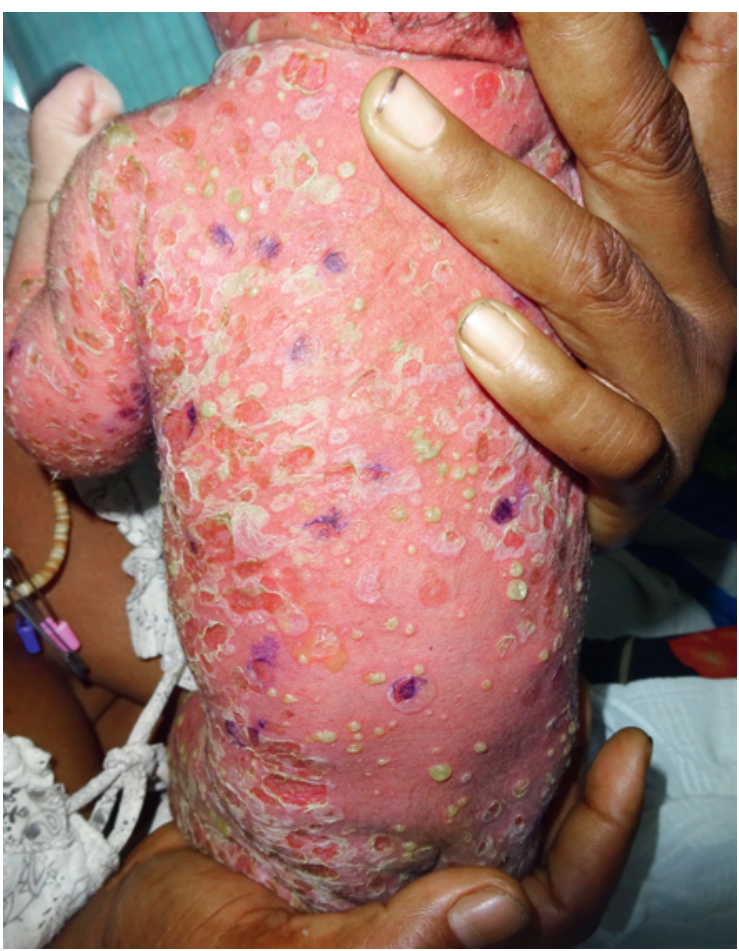

Abbildung 2: Generalisierte Staphylokokken-Pustulose bei einem drei Tage alten Kind.

\section{Das Erdbeben}

Am 9. Dezember 2016 morgens um 4:38 wurden wir durch ein Erdbeben der Stärke 7,8 aus dem Bett geschüttelt. Mit Apotheke, Trinkwasserfilter, Moskitospray, Wasser und Schokolade bewaffnet und der Stirnlampe auf dem Kopf verliess ich torkelnd das immer noch bebende Haus. Wir suchten einen Weg im Busch, um auf einen Hügel zu gelangen, um dem möglichen Tsunami zu entkommen. Durch ein rauschendes Radio erhielten wir erste Informationen und erfuhren wir auch, dass es bis jetzt keine Berichte über Verletzte gab. Das war für mich beruhigend, da die beiden anderen Ärzte am Nachmittag in die Ferien in eine andere Provinz flogen. Damit war ich die einzige Ärztin in der ganzen Provinz. Im Busch traf ich auch auf alle meine Patienten aus dem Spital. Diejenigen, die nicht laufen konnten, wurden auf Bahren hinaufgetragen. Zum Glück wurde der letzte wirklich akute Patient am Vortag mit dem Flugzeug ausgeflogen. So machte ich meine morgendliche Visite unter freiem Himmel. Ich checkte die mangelernährten Kinder und schaute, dass diese regelmässig gestillt wurden, da die Ergänzungsmilch im Spital zurückgeblieben war, und dass die Dengue-Patienten, die ihre Infusion verloren hatten, genügend Wasser tranken. Später verteilte einer der Pfleger auch Medikamente, welche ich dann durch meine eigenen ergänzte, da nicht alles vorhanden war.
Nach 5 Stunden ging ich zurück ins Dorf. Trotz viertelstündlichen Nachbeben der Stärke 5. Das Spital war verlassen, kein einziger Patient war anzutreffen. Einige herausgerissene Infusionen lagen noch herum. Glücklicherweise überlebten das Ultraschall- und Röntgengerät, im Gegensatz zu den meisten Computern. Auch hatten wir Glück, dass keine der umgefallenen Sauerstoffflaschen explodiert war.

Am späteren Abend waren dann die meisten Patienten wieder zurück und ich konnte die Visite nachholen. Auch 3 der 10 für diesen Tag aufgebotenen Patienten tauchten auf.

\section{Fazit}

In diesen 4 Wochen lernte ich enorm viel. Nur selten konnte ich jemandem Fragen stellen und ich hatte sehr beschränkte diagnostische Mittel (kleines Blutbild, Malaria- und Dengue-Test, Röntgen, Ultraschall). So brauchte es manchmal Fantasie, Improvisationsvermögen und handwerkliches Geschick, um etwas therapieren zu können. Doch gerade das ist es, was mich reizt, erneut zurückzukehren. Ein Land, wo man mit wenig auskommen muss, aber trotzdem viel bewirken kann. Ein Land, in dem die Patienten dankbarer sind und auch einige Stunden Wartezeit ohne Murren in Kauf nehmen. Etwas dieser Gelassenheit würde uns auch in der Schweiz gut tun.

So verliess ich nun einen Tag nach dem Erdbeben das Spital und die Provinz. Mit mir flogen zwei Patienten, die ich ins NRH überbrachte. Ich verliess ein Spital, das

\section{Die Arbeit war sehr abwechslungsreich und auch herausfordernd.}

nach dem Erdbeben noch intakt und funktionsfähig war, jedoch für einen guten Monat ohne Arzt auskommen muss!

\section{Dank an}

Dr. Grace Rara und Dr. Arnold Nguduame, sowie die Mitarbeiter des Kirakira Hospitals; die Mitarbeiter der Fracture Clinic im NRH, insb. Dr. Patrick Houasia; Dr. Dr. Hermann Oberli, der mich mit den Salomonen «angesteckt» hat.

\section{Bildnachweise}

Helen Laura Moser

\section{Haben auch Sie Interesse an einem}

\section{Einsatz im Südpazifik?}

Ärzte/Studenten können sich gern auf der Seite vom Verein «Medizin im Südpazifik» auf www.hermannoberli.ch über einen möglichen Einsatz informieren. 